\title{
Universal nonlinear infection kernel from heterogeneous exposure on higher-order networks
}

\author{
Guillaume St-Onge, ${ }^{1,2, *}$ Hanlin Sun, ${ }^{3}$ Antoine Allard, ${ }^{1,2,4}$ Laurent Hébert-Dufresne, ${ }^{1,4,5}$ and Ginestra Bianconi ${ }^{3,6}$ \\ ${ }^{1}$ Département de physique, de génie physique et d'optique, \\ Université Laval, Québec (Québec), Canada G1V OA6 \\ ${ }^{2}$ Centre interdisciplinaire en modélisation mathématique, \\ Université Laval, Québec (Québec), Canada G1V OA6 \\ ${ }^{3}$ School of Mathematical Sciences, Queen Mary University of London, London, E1 4NS, United Kingdom \\ ${ }^{4}$ Vermont Complex Systems Center, University of Vermont, Burlington, VT 05405 \\ ${ }^{5}$ Department of Computer Science, University of Vermont, Burlington, VT 05405 \\ ${ }^{6}$ The Alan Turing Institute, 96 Euston Rd, London NW1 2DB, United Kingdom
}

\begin{abstract}
The colocation of individuals in different environments is an important prerequisite for exposure to infectious diseases on a social network. Standard epidemic models fail to capture the potential complexity of this scenario by (1) neglecting the higher-order structure of contacts which typically occur through environments like workplaces, restaurants, and households; and by (2) assuming a linear relationship between the exposure to infected contacts and the risk of infection. Here, we leverage a hypergraph model to embrace the heterogeneity of environments and the heterogeneity of individual participation in these environments. We find that combining heterogeneous exposure with the concept of minimal infective dose induces a universal nonlinear relationship between infected contacts and infection risk. Under nonlinear infection kernels, conventional epidemic wisdom breaks down with the emergence of discontinuous transitions, super-exponential spread, and hysteresis.
\end{abstract}

Mathematical models of epidemics play an increasingly important role in public health efforts and pandemic preparedness [1]. By providing insights on the interplay of the biological and sociological aspects of epidemics, models can test potential interventions in silico and suggest potential outcomes [2]. However, large-scale forecasting comparisons show that statistical models often outperform mechanistic models that make assumptions about spreading dynamics [3].

In this letter, we look at the interplay of two commonly used assumptions in disease models: Random mixing and the linearity between infection risk and exposures to infected individuals. In almost all disease models, doubling the number of contacts between susceptible and infectious individuals doubles the risk of infection for the susceptible individuals. Some past work in mathematical biology has considered nonlinear infection rates $[4,5]$, but these models are rarely used in practice. Other fields such as sociology do often consider generalized contagion models, often dubbed complex contagions [6, 7]. In these complex contagions, having a nonlinear relationship between infection rate and sources of infection allows the model to consider mechanisms such as social reinforcement [8], where a set of multiple exposures can have more impact than the mere sum of unique exposures.

The mathematical convenience of assuming random mixing when modeling infectious diseases comes at the price that all contacts between susceptible and infectious individuals are effectively equivalent. This assumption has often been lifted using heterogeneous mathematical models where individuals are distinguished by some individual features such as their intrinsic susceptibility or reaction to the infection $[9,10]$, relaxing the mass-action assumption directly $[11,12]$, or by specifying an underlying contact network [13, 14].

Moreover, the linearity assumption says that all increments in the total exposure to infectious individuals (measured for example as a viral inoculum) are equivalent. Evidence asso- ciated with the minimal infective dose of different infectious diseases shows that not all exposures are equal, and that some minimal dose might be required for an infection to likely occur. More precisely, the ID $_{50}$ value is a measure of the dose needed to cause an infection in $50 \%$ of individuals. These concepts are needed because our immune system is usually able to handle microscopic challenges from viruses and bacteria alike. While an infective dose of tuberculosis might only require between 1 and 5 bacteria [19], some enterics might require up to $10^{9}$ pathogenic particles [20], and others like common respiratory infections still require further study [21]. There are indeed multiple different physical mechanisms behind immune evasion, for example some airborne viruses need to find their receptors on lung epithelial cells, while some bacteria might instead require interaction with the immune system [22]. These mechanisms are reviewed in Refs. [22-26], and all of them combine to determine the ID50 of specific pathogens. Likewise, the decay or clearing rates of pathogens in non-infectious courses can also vary a lot, potentially requiring days for bacteria to hours for airborne viral infections. For example, mathematical models for the pathogenesis of SARS-CoV-2 or influenza A use decay rates of the order of 7-18 hours but empirical estimates vary wildly (see Ref. [27] and [28] and references therein).

To study the effect of simultaneously relaxing these two assumptions, we consider a social structure where individuals attend a certain number of environments such as work places, gyms, or supermarkets. This division of contact structure in environments is motivated by the known role of superspreading events, which are for example critical to the ongoing spread of COVID-19 [29-36]. While variations at the individual level is often used to explain superspreading [37], we focus here on the variability of environments and of temporal patterns [38-44] at the group level, which undoubtedly affect epidemics [31], especially when a certain exposure within a 

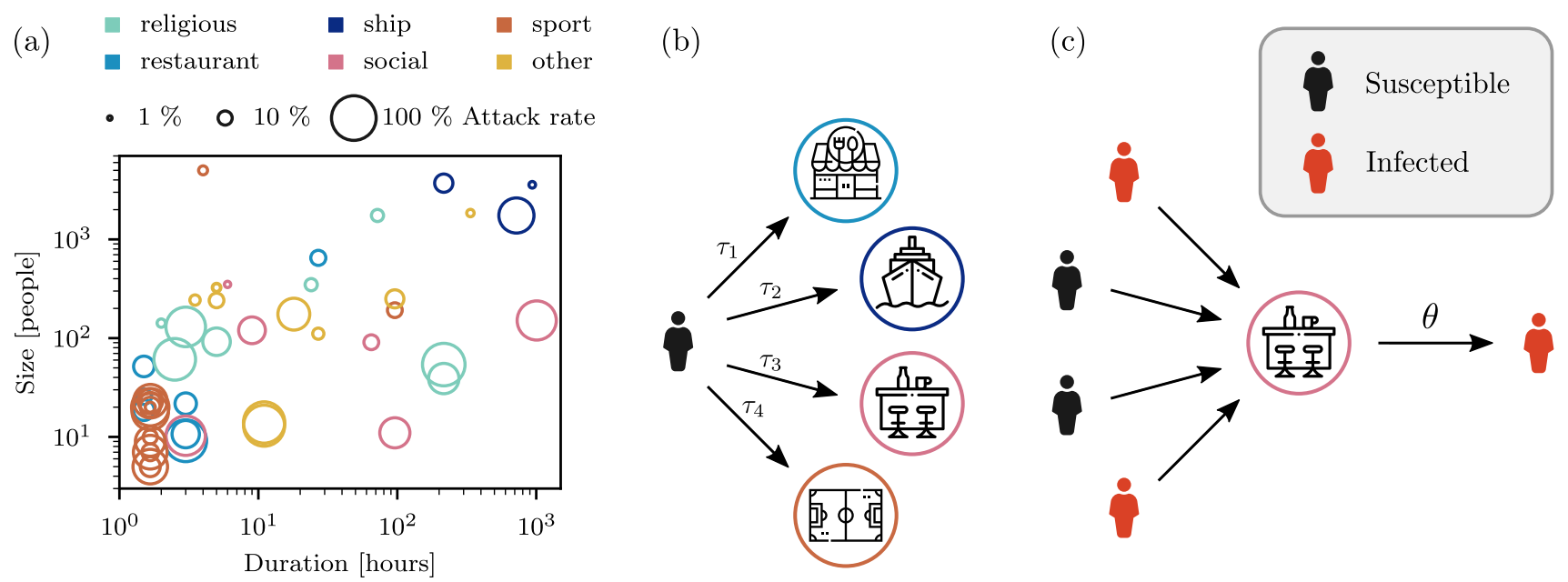

FIG. 1. Modeling contagions and superspreading events through higher-order networks. (a) Scatter plot of superspreading events of COVID-19 where the number of people involved (size), the duration of the event, and the resulting proportion of infected individuals (attack rate) are all available (extracted from Refs. [15, 16], see the supplemental material [17]). (b)-(c) Framework for contagions on hypergraphs [18], where the size $m$ of the hyperedges (environment), the hyperdegree $k$ of the nodes (individuals), and the participation time to the environment $\tau$ are all heterogeneous, distributed according to $\hat{P}(m), \tilde{P}(k)$, and $P(\tau)$ respectively. For the sake of simplicity, we assume the same distribution $P(\tau)$ for all environments. (b) At each time step $t$, an individual participates for a time $\tau$ (drawn independently) to each environment. (c) An individual gets infected with probability $\theta_{m}(\rho)$ in the environment at time step $t$, which depends on the size $m$ and the fraction infected $\rho$.

certain time window is needed to confidently spark an infection. Interestingly, available case data highlight how there is no expected size or duration for such events. Transmission is highly context dependent on the settings (e.g., ventilation) and activity (e.g., singing or shouting) such that the resulting superspreading events are heterogeneous in size, duration and attack rate, as shown in Fig. 1(a). Higher-order contact structures and heterogeneous temporal patterns are therefore key ingredients for more realistic models of spreading dynamics.

Mathematically, we represent the contact structure as a hypergraph [45-47] where each environment is described by a hyperedge connecting $m$ nodes (individuals) and where each node is incident to $k$ hyperedges. All hyperedges of a same size $m$ are considered equivalent, although this assumption is relaxed in the supplemental material to consider additional sources of heterogeneity [17]. To model heterogeneous temporal patterns, we consider a discrete-time process, where at each time step $t=1,2, \ldots$, we draw for each individual a participation time $\tau \in\left[1, \tau_{\max }\right]$ for each environment to which they are connected [Fig 1(b)]. The time steps correspond to fixed temporal windows of size $\tau_{\max }$, during which susceptible individuals can get infected through their participation to environments.

We first study the impact of the spatiotemporal co-location patterns on the infection kernel $\theta_{m}(\rho)$, the probability of getting infected in an environment of size $m$ when a fraction $\rho$ of the other participants are infectious [Fig. 1(c)]. We then analyze the properties of the resulting contagion process.

Universal infection kernel from heterogeneous exposure. Let us consider a susceptible individual participating to an environment of size $m$ for a duration $\tau$, where a fraction $\rho$ of the other participants are infectious. During this exposure period, some of the other $m-1$ individuals might participate to the environment as well. We assume that the considered individual receives an infective dose $\kappa \in[0, \infty)$ from the infectious individuals, distributed according to $\pi(\kappa ; \lambda)$, where $\lambda \equiv\langle\kappa\rangle$. A reasonable assumption is that the mean dose received is proportional to the time spent in the environment and to the proportion of infectious people, $\lambda=\beta f(m) \tau \rho$, where $\beta$ is a rate of dose accumulation and $f(m)$, unitless, modulates the typical number of contacts in environments frequented by $m$ individuals.

While this is not a strict requirement for our results to hold (see supplemental material [17]), we further assume that the random variable for the dose can be written as $\kappa=\lambda u$, where $u$ is a random variable that is independent of $\lambda$. In this case, $u$ can be seen as an intrinsic property of the contagion processdetermined by rates of viral shedding, diffusion in the environment, variability of human interactions, etc.-while $\lambda$ acts as a scale parameter, i.e., $\pi(\kappa ; \lambda)=\pi(\kappa / \lambda ; 1) / \lambda \equiv \pi(\kappa / \lambda) / \lambda$.

To incorporate the concept of minimal infective dose, we assume that an individual develops the disease if $\kappa>K$, a perspective analogous to standard threshold models [48-50] and related to the assumption that successful host invasion necessitates multiple attempts by the pathogen [51]. The probability of getting infected in the environment is then

$$
\bar{\Pi}(K / \lambda)=\int_{K / \lambda}^{\infty} \pi(\kappa) \mathrm{d} \kappa .
$$

The infection kernel $\theta_{m}(\rho)$ is calculated by averaging $\bar{\Pi}(K / \lambda)$ over $P(\tau)$. We focus here on the case of heterogeneous exposure periods modeled with a Pareto distribution $P(\tau)=C_{\alpha} \tau^{-\alpha-1}$, where $C_{\alpha}$ is a normalization constant, $\alpha>0$ and $\tau \in\left[1, \tau_{\max }\right]$. However, for our dose mechanism to be 
well defined, we can only average over participation times $\tau \in[1, \mathcal{T}]$, where $\mathcal{T} \leq \tau_{\max }$ is the clearing window, i.e., the characteristic time for the immune system to get rid of any dose $\kappa<K$. If we assume that this clearing window is sufficiently large compared to $\tau_{\mathrm{c}} \equiv K / \beta f(m) \rho$, the characteristic time to get infected in the environment, we can neglect events where $\tau \geq \mathcal{T}$ as they do not contribute significantly to the infection kernel [17]. We therefore redefine our support as $\tau \in[1, \mathcal{T}]$ and the infection kernel is

$$
\begin{aligned}
\theta_{m}(\rho) & =\int_{1}^{\mathcal{T}} \bar{\Pi}\left(\tau_{\mathrm{c}} / \tau\right) P(\tau) \mathrm{d} \tau \\
& =\frac{C_{\alpha}}{\alpha}\left[\bar{\Pi}\left(\tau_{\mathrm{c}}\right)-\bar{\Pi}\left(\tau_{\mathrm{c}} / \mathcal{T}\right) \mathcal{T}^{-\alpha}+\tau_{\mathrm{c}}^{-\alpha} \int_{\tau_{\mathrm{c}} / \mathcal{T}}^{\tau_{\mathrm{c}}} \pi(y) y^{\alpha} \mathrm{d} y\right]
\end{aligned}
$$

When $1 \ll \tau_{\mathrm{c}} \ll \mathcal{T}$ and $\pi(y)$ decreases faster than $y^{-\alpha-1}$, then the integral on the right converges to a constant, the term in $\mathcal{T}^{-\alpha}$ can be neglected, and $\bar{\Pi}\left(\tau_{\mathrm{c}}\right) \ll \tau_{\mathrm{c}}^{-\alpha}$, which implies

$$
\theta_{m}(\rho) \sim D_{\alpha} \tau_{\mathrm{c}}^{-\alpha} \propto \rho^{\alpha},
$$

where $D_{\alpha}$ is some constant. This form of infection kernel is universal, driven by temporal patterns, and does not depend on the value of $K$ (given $K>0$ ) or on the particular form of $\pi$. We illustrate it in Fig. 2(a) using an exponential for the dose distribution-other cases such as the Weibull and the Fréchet distribution are presented in the supplemental material [17].

Let us stress that the condition $1 \ll \tau_{\mathrm{c}} \ll \mathcal{T}$ is not restrictive. On the time scale on which we measure the exposure periods, $\tau_{\mathrm{c}} \gg 1$ implies that the contagion does not spread too fast, otherwise the whole population would be rapidly infected, while $\mathcal{T} \gg \tau_{\mathrm{c}}$ suggests that it is transmissible before the immune system is able to clear the dose received.

More broadly, we do not need to assume that $\pi(y)$ decreases faster than $y^{-\alpha-1}$, nor that $\lambda$ is a scale parameter for $\kappa$. In fact, $\pi(\kappa ; \lambda)$ does not even need to be a continuous distribution and $P(\tau)$ could be asymptotically power law for large $\tau$. In this more general context, we still recover a universal infection kernel $\theta_{m}(\rho) \propto \rho^{v}$ in most cases, but $v$ is not always directly equal to $\alpha$ [17]. Linear infection kernels $(v=1)$ are recovered only in some specific cases, for instance when $\alpha=1$ or when we use a Poisson distribution for $\kappa$ and $K=1$. From now on, we make abstraction of the underlying distributions $\pi(\kappa ; \lambda)$ and $P(\tau)$, and focus on the resulting effective model parametrized by $v$.

Epidemic spreading with nonlinear infection kernel. We now illustrate the consequences of nonlinear infection kernels for contagion on hypergraphs. To simplify the mathematical analysis, we consider a Susceptible-Infectious-Susceptible model. At each time step, an infected node becomes susceptible with probability $\mu$, while a susceptible node gets infected through a hyperedge of size $m$ with probability $\theta_{m}$. If a node is part of multiple hyperedges, we assume that the probability of infection through each hyperedge are independent. This is reasonable if $k_{\max } \mathcal{T} \leq \tau_{\max }$ for a maximal hyperdegree $k_{\max }$. For instance, if $\mathcal{T}$ is of the order of a few hours, $\tau_{\max }$ a week, and an individual participate to an environment once a day, the night allows the immune system to clear any dose $\kappa<K$ accumulated the day before.

To obtain some analytical insights, we introduce a degreebased mean-field theory [14], an approximation equivalent to consider an infinite size annealed hypergraph, where at each time step, the connections between nodes and hyperedges are shuffled. We validate our approach with Monte Carlo simulations on quenched hypergraphs in the supplemental material [17].

With the mean-field approximation, the marginal probability for a node to be infected at time $t$ only depends on its hyperdegree, which we note $\rho_{k}(t)$. The global prevalence is then simply $I(t)=\sum_{k} \rho_{k}(t) \tilde{P}(k)$ and the evolution of the system is described by [14]

$$
\rho_{k}(t+1)=(1-\mu) \rho_{k}(t)+\left[1-\rho_{k}(t)\right] \Theta_{k}(\bar{\rho}),
$$

where $\Theta_{k}(\bar{\rho})=1-[1-\bar{\theta}(\bar{\rho})]^{k}$ is the probability for a susceptible node of hyperdegree $k$ to get infected. $\bar{\rho}(t)$ is the probability that a node belonging in any hyperedge is infected and $\bar{\theta}(\bar{\rho})$ is the probability for a susceptible node to get infected in any hyperedge,

$$
\bar{\rho}(t)=\sum_{k} \rho_{k}(t) \frac{k \tilde{P}(k)}{\langle k\rangle} \text { and } \bar{\theta}(\bar{\rho})=\sum_{m} \bar{\theta}_{m}(\bar{\rho}) \frac{m \hat{P}(m)}{\langle m\rangle},
$$

where $\bar{\theta}_{m}(\bar{\rho})$ is the probability for a node to get infected in a hyperedge of size $m$. Because of the annealed structure, $\bar{\theta}_{m}(\bar{\rho})$ is just the average of $\theta_{m}(\rho)$ with $\rho=i /(m-1)$ over a binomial distribution,

$$
\bar{\theta}_{m}(\bar{\rho})=\sum_{i=0}^{m-1}\left(\begin{array}{c}
m-1 \\
i
\end{array}\right) \bar{\rho}^{i}(1-\bar{\rho})^{m-1-i} \theta_{m}\left(\frac{i}{m-1}\right),
$$

with $\theta_{m}(\rho)$ defined at Eq. (2).

Figure 2(b) shows a first consequence of the nonlinear kernel: the apparition of super-exponential growth for the global prevalence $I(t)$ when $v>1$. Note that the growth is approximately exponential until a sufficiently high prevalence. For $v \leq 1$, we instead have a standard exponential growth until saturation is reached.

In the steady state of the epidemic dynamics, we obtain a self-consistent solution

$$
\rho_{k}^{*}=\frac{\Theta_{k}^{*}}{\mu+\Theta_{k}^{*}} \quad \text { and } \quad \bar{\rho}^{*}=\sum_{k} \tilde{P}(k) \frac{k}{\langle k\rangle} \frac{\Theta_{k}^{*}}{\mu+\Theta_{k}^{*}} \equiv G\left(\bar{\rho}^{*}\right),
$$

since $\Theta_{k}$ is a function of $\bar{\rho}$.

For contagions with a nonlinear infection kernel, the phase transition associated with the order parameter $I^{*}$ can be continuous or discontinuous with a bistable regime. Consequently, we define the invasion threshold $\beta_{\mathrm{c}}$ such that for all $\beta>\beta_{\mathrm{c}}$, the absorbing state $I^{*}=0$ is unstable [dashed line in Fig. 2(c)]. We also define the persistence threshold $\beta_{\mathrm{p}}$ such that for all $\beta<\beta_{\mathrm{p}}$, the absorbing state $I^{*}=0$ is globally attractive [dotted line in Fig. 2(c)]. For continuous phase transitions, $\beta_{\mathrm{c}}$ and $\beta_{\mathrm{p}}$ coincide, and is called the epidemic threshold; for a discontinuous phase transition, $\beta_{\mathrm{p}}<\beta_{\mathrm{c}}$, and for all 
(a) Infection kernel

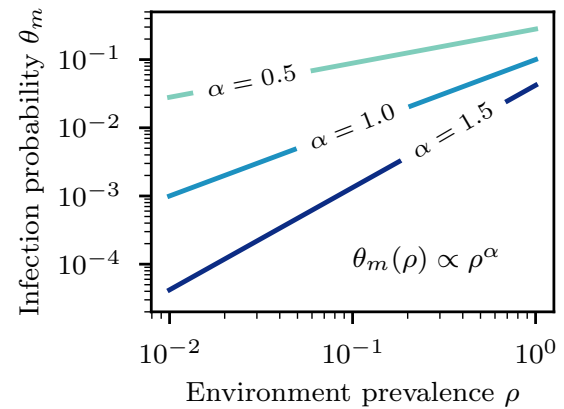

(b) Temporal evolution

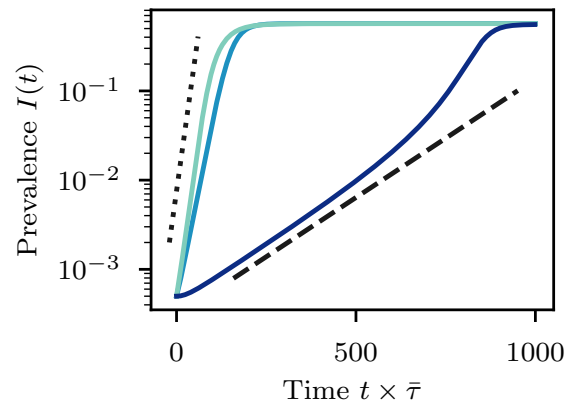

(c) Phase diagram

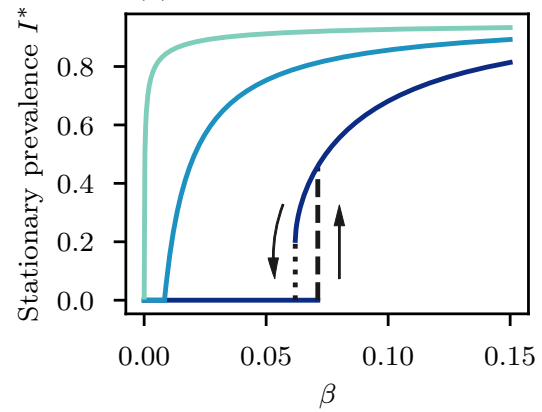

FIG. 2. Properties of contagions with nonlinear infection kernels induced by heterogenous exposure. We use an exponential dose distribution $\pi(\kappa ; \lambda) \propto e^{-\kappa / \lambda}$ with a power-law distribution of participation time $P(\tau) \propto \tau^{-\alpha-1}$, a clearing window $\mathcal{T} \rightarrow \infty$, and $f(m)=1$. (a) Effective infection kernel using $\beta=0.1$. The infection probability has a power law scaling $\theta_{m}(\rho) \propto \rho^{\alpha}$. (b)-(c) We use Poisson distributions for both $\tilde{P}(k)$ and $\hat{P}(m)$, with $\langle k\rangle=5$ and $\langle m\rangle=10$, and set $\mu=0.05$. We use Eqs. (4-6) to evolve the system. (b) Supra-linear kernels $v>1$ lead to a super-exponential growth for the global prevalence $I(t)$. We use $\beta=5 \times 10^{-4}, \beta=0.025$ and $\beta=0.077$ for $v=0.5, v=1$ and $v=1.5$ respectively. $\bar{\tau}$ is the median exposure period. (c) The phase diagram for stable solutions in the stationary state $(t \rightarrow \infty)$ can be continuous or discontinuous with a bistable regime. Sub-linear and linear kernels $v \leq 1$ lead to a continuous phase transition, and the invasion threshold $\beta_{\mathrm{c}}$ vanishes for $v \rightarrow 0$. Supra-linear kernels $v>1$ can lead to a discontinuous phase transition with a bistable regime.

$\beta \in\left(\beta_{\mathrm{p}}, \beta_{\mathrm{c}}\right)$, there exists typically three solutions, $I_{1}^{*}=0$ and $I_{2}^{*}, I_{3}^{*}>0$, with $I_{1}^{*}$ and $I_{3}^{*}$ locally stable.

The invasion threshold $\beta_{\mathrm{c}}$ can be found by imposing $G^{\prime}(0)=1$, the persistence threshold $\beta_{\mathrm{p}}$ is obtained by imposing both $\bar{\rho}^{*}=G\left(\bar{\rho}^{*}\right)$ and $G^{\prime}\left(\bar{\rho}^{*}\right)=1$ for $\bar{\rho}^{*}>0$, and any tricritical point can be found by imposing $G^{\prime}(0)=1$ and $G^{\prime \prime}(0)=0$.

In the supplemental material [17], we obtain an exact selfconsistent expression for the invasion threshold, and using again an asymptotic approximation, we find

$$
\beta_{\mathrm{c}} \propto\left(\frac{\mu\langle m\rangle\langle k\rangle}{\left\langle m(m-1)^{1-v}[f(m)]^{\nu}\right\rangle\left\langle k^{2}\right\rangle}\right)^{1 / v} .
$$

It depends in a intricate manner on both the moments of $\hat{P}(m)$ and $\tilde{P}(k)$ and the nonlinearity of the infection kernel. As illustrated in Fig. 2(c), the invasion threshold can become very small for $v<1$ (vanishing for $v \rightarrow 0$ ), even for homogeneous $\tilde{P}(k)$ and $\hat{P}(m)$. Note that to obtain a sub-linear kernel $v<1$, it typically requires a distribution $P(\tau) \propto \tau^{-\alpha-1}$ with $\alpha<1$, which implies that the mean participation time $\langle\tau\rangle$ diverges. In the supplemental material [17], we show that if instead we fix $\langle\tau\rangle$ while varying $\alpha$, there exists an optimal temporal heterogeneity $\alpha^{*}$ that minimizes the invasion threshold $\beta_{\mathrm{c}}$, and maximizes early spread.

The minimal kernel exponent $v_{\mathrm{c}}$ leading to a discontinuous phase transition is given by a tricritical point [17]. Although exact solutions require numerical evaluation, we get three insights from an asymptotic expansion. (i) $v>1$ is necessary in order to have a discontinuous phase transition, but it is not sufficient: $v_{\mathrm{c}}$ depends on the first three moments of $\tilde{P}(k)$, and in a more complicated manner on the distribution $\hat{P}(m)$. (ii) It is necessary to have environments of size $m>2$ to have a discontinuous phase transition. (iii) A more heterogeneous $\tilde{P}(k)$ leads to a larger $v_{\mathrm{c}}$. Similar observations were made in Ref. [52] for $m \leq 3$.

Conclusion. Our framework captures many properties usually overlooked for the sake of simplicity in epidemic models: the higher-order structure of contacts, the temporal heterogeneity of human activity and thresholding effects over the exposure due to the host immune system. In the supplemental material [17], we also demonstrate that our results are robust to variations in individual infectiousness or local transmission in different environments. In particular, we recover a universal nonlinear infection kernel that provides a connection between complex contagions based on nonlinear infection kernels [53] and threshold models [48-50].

Our results challenge a key assumption of most epidemic models and ask: Why assume a linear relationship between the number of infectious contacts and the risk of infection? This question is critical since three of the basic insights gathered from epidemic models break down under nonlinear infection kernels: they can lead to a discontinuous relationship between disease transmission and epidemic size, to a bistable regime where macroscopic outbreak and disease-free state coexist, and to a super-exponential growth. While the first two are difficult to assess for real contagions, super-exponential spread has been observed for influenza-like illness [54].

Even though we considered the SIS model to simplify the analysis, the universal infection kernel $\theta_{m}(\rho) \propto \rho^{v}$ could be directly integrated in more realistic models-such as SEIR or SIRS — where the same phenomenology typically carries over.

The phenomenology being drastically different from standard epidemiological models begs the following question: Why do linear models work? Even for a nonlinear kernel $\theta_{m}(\rho)$, the probability of infection $\bar{\theta}_{m}(\bar{\rho})$ (averaged over hyperedge configurations) is linear in $\bar{\rho}$ if $\bar{\rho} \ll 1$ [see Eq. (6)]. Therefore, linear models are a good approximation when the prevalence is sufficiently low, but breaks down at higher prevalence, as clearly illustrated in Fig. 2(b) when $v=1.5$.

The mathematical framework we use to solve the SIS model hinges on a mean-field or annealed approximation, as in other studies [52, 55-57], thereby suppressing dynamical correlations within hyperedges. As we show in the supplemental 
material [17], dynamical correlations can be captured using approximate master equations [58-64], which are more complicated but provide similar results with better agreement to simulations. Future works could investigate more thoroughly the interplay between dynamical correlations, nonlinear kernels, and spatiotemporal heterogeneity.

Altogether, our conclusions stress the need to embrace heterogeneity in disease modeling; in the infection dynamics itself, in patterns of temporal activity, and in the higher-order structure of contact networks. Epidemics should be seen as the result of a collective process, where higher-order structure and temporal patterns can drive complex dynamics.

The authors thank Dr. Sean Diehl for help with advanced immunological concepts. This work was supported by the Fonds de recherche du Québec - Nature et technologies (G.S.), the Natural Sciences and Engineering Research Council of Canada (G.S., A.A.), the Sentinelle Nord program from the Canada First Research Excellence Fund (G.S., A.A.), the Chinese Scholarship Council (H.S.), the National Institutes of Health 1P20 GM125498-01 Centers of Biomedical Research Excellence Award (L.H.-D.), the Royal Society (IEC $\backslash N S F C \backslash 191147$; G.B.).

*guillaume.st-onge.4@ulaval.ca

[1] C. Rivers, J.-P. Chretien, S. Riley, J. A. Pavlin, A. Woodward, D. Brett-Major, I. Maljkovic Berry, L. Morton, R. G. Jarman, M. Biggerstaff, M. A. Johansson, N. G. Reich, D. Meyer, M. R. Snyder, and S. Pollett, Using "outbreak science" to strengthen the use of models during epidemics, Nat. Commun. 10, 3102 (2019).

[2] J. M. Epstein, Why Model?, J. Artif. Soc. Soc. Simul. 11, 12 (2008).

[3] M. Biggerstaff, M. Johansson, D. Alper, L. C. Brooks, P. Chakraborty, D. C. Farrow, S. Hyun, S. Kandula, C. McGowan, N. Ramakrishnan, R. Rosenfeld, J. Shaman, R. Tibshirani, R. J. Tibshirani, A. Vespignani, W. Yang, Q. Zhang, and C. Reed, Results from the second year of a collaborative effort to forecast influenza seasons in the United States, Epidemics 24, 26 (2018).

[4] W.-m. Liu, S. A. Levin, and Y. Iwasa, Influence of nonlinear incidence rates upon the behavior of SIRS epidemiological models, J. Math. Biol. 23, 187 (1986).

[5] H. W. Hethcote and P. van den Driessche, Some epidemiological models with nonlinear incidence, J. Math. Biol. 29, 271 (1991).

[6] D. Centola, How Behavior Spreads: The Science of Complex Contagions (Princeton University Press, 2018).

[7] S. Lehmann and Y.-. Ahn, Complex Spreading Phenomena in Social Systems (Springer, 2018).

[8] D. Centola, The Spread of Behavior in an Online Social Network Experiment, Science 329, 1194 (2010).

[9] S. N. Busenberg, M. Iannelli, and H. R. Thieme, Global Behavior of an Age-Structured Epidemic Model, SIAM J. Math. Anal. 22, 1065 (1991).

[10] Z. Feng and H. R. Thieme, Endemic Models with Arbitrarily Distributed Periods of Infection I: Fundamental Properties of the Model, SIAM J. Appl. Math. 61, 803 (2000).
[11] H. W. Hethcote and J. W. Van Ark, Epidemiological models for heterogeneous populations: Proportionate mixing, parameter estimation, and immunization programs, Math. Biosci. 84, 85 (1987).

[12] H. W. Hethcote, Modeling heterogeneous mixing in infectious disease dynamics, in Models for Infectious Human Diseases: Their Structure and Relation to Data (Cambridge University Press, 1996) 1st ed., pp. 215-238.

[13] R. Pastor-Satorras and A. Vespignani, Epidemic Spreading in Scale-Free Networks, Phys. Rev. Lett. 86, 3200 (2001).

[14] R. Pastor-Satorras, C. Castellano, P. Van Mieghem, and A. Vespignani, Epidemic processes in complex networks, Rev. Mod. Phys. 87, 925 (2015).

[15] K. Swinkels, SARS-CoV-2 superspreading events from around the world (2020), accessed: 2020-07-02.

[16] Q. J. Leclerc, N. M. Fuller, L. E. Knight, S. Funk, G. M. Knight, and C. C.-. W. Group, What settings have been linked to sars-cov-2 transmission clusters?, Wellcome Open Research 5 (2020).

[17] See Supplemental Material for a broader derivation of the infection kernel, the consideration of other sources of heterogeneity, the characterization of the phase transition, and a treatment of dynamical correlations, which includes Refs. [15, 16, 63-70].

[18] Icons made by Freepik from www.flaticon.com.

[19] V. Balasubramanian, E. H. Wiegeshaus, B. T. Taylor, and D. W. Smith, Pathogenesis of tuberculosis: Pathway to apical localization, Tuber. Lung Dis. 75, 168 (1994).

[20] R. C. LaRocque and S. B. Calderwood, Syndromes of enteric infection, in Mandell, Douglas, and Bennett's Principles and Practice of Infectious Diseases (Elsevier, 2015) pp. 1238-1247.

[21] T. P. Weber and N. I. Stilianakis, Inactivation of influenza A viruses in the environment and modes of transmission: A critical review, J. Infect. 57, 361 (2008).

[22] J. A. Gama, S. S. Abby, S. Vieira-Silva, F. Dionisio, and E. P. C. Rocha, Immune subversion and quorum-sensing shape the variation in infectious dose among bacterial pathogens, PLOS Pathog. 8, e1002503 (2012).

[23] B. B. Finlay and S. Falkow, Common themes in microbial pathogenicity revisited., Microbiol. Mol. Biol. Rev. 61, 136 (1997).

[24] M. W. Hornef, M. J. Wick, M. Rhen, and S. Normark, Bacterial strategies for overcoming host innate and adaptive immune responses, Nat. immunol. 3, 1033 (2002).

[25] M. Lipsitch and J. J. O'Hagan, Patterns of antigenic diversity and the mechanisms that maintain them, J. R. Soc. Interface 4, 787 (2007).

[26] A. Casadevall, Evolution of intracellular pathogens, Annu. Rev. Microbiol. 62, 19 (2008).

[27] S. Q. Du and W. Yuan, Mathematical modeling of interaction between innate and adaptive immune responses in COVID-19 and implications for viral pathogenesis, J. Med. Virol. 92, 1615 (2020).

[28] C. A. Beauchemin and A. Handel, A review of mathematical models of influenza A infections within a host or cell culture: lessons learned and challenges ahead, BMC Public Health 11, S7 (2011).

[29] Y. Liu, R. M. Eggo, and A. J. Kucharski, Secondary attack rate and superspreading events for sars-cov-2, Lancet 395, e47 (2020).

[30] F. Wong and J. J. Collins, Evidence that coronavirus superspreading is fat-tailed, Proc. Natl. Acad. Sci. U.S.A. 117, 29416 (2020).

[31] B. M. Althouse, E. A. Wenger, J. C. Miller, S. V. Scarpino, A. Allard, L. Hébert-Dufresne, and H. Hu, Superspreading 
events in the transmission dynamics of SARS-CoV-2: Opportunities for interventions and control, PLOS Biol. 18, e3000897 (2020).

[32] A. Endo, S. Abbott, A. J. Kucharski, and S. Funk, Estimating the overdispersion in COVID-19 transmission using outbreak sizes outside china, Wellcome Open Res. 5, 67 (2020).

[33] Q. Bi et al., Epidemiology and transmission of COVID-19 in 391 cases and 1286 of their close contacts in shenzhen, china: a retrospective cohort study, Lancet Infect. Dis. 20, 911 (2020).

[34] D. Miller, M. A. Martin, N. Harel, O. Tirosh, T. Kustin, M. Meir, N. Sorek, S. Gefen-Halevi, S. Amit, O. Vorontsov, et al., Full genome viral sequences inform patterns of SARSCoV-2 spread into and within israel, Nat. Commun. 11, 5518 (2020).

[35] M. S. Y. Lau, B. Grenfell, M. Thomas, M. Bryan, K. Nelson, and B. Lopman, Characterizing superspreading events and agespecific infectiousness of SARS-CoV-2 transmission in Georgia, USA, Proc. Natl. Acad. Sci. U.S.A. 117, 22430 (2020), https://www.pnas.org/content/117/36/22430.full.pdf.

[36] B. F. Nielsen, L. Simonsen, and K. Sneppen, Covid-19 superspreading suggests mitigation by social network modulation, Phys. Rev. Lett. 126, 118301 (2021).

[37] J. O. Lloyd-Smith, S. J. Schreiber, P. E. Kopp, and W. M. Getz, Superspreading and the effect of individual variation on disease emergence, Nature 438, 355 (2005).

[38] M. Karsai, H.-H. Jo, and K. Kaski, Bursty Human Dynamics (Springer International Publishing, 2018).

[39] M. Karsai, K. Kaski, A.-L. Barabási, and J. Kertész, Universal features of correlated bursty behaviour, Sci. Rep. 2, 397 (2012).

[40] P. Holme and J. Saramäki, Temporal networks, Phys. Rep. 519, 97 (2012).

[41] C. Cattuto, W. Van den Broeck, A. Barrat, V. Colizza, J.-F. Pinton, and A. Vespignani, Dynamics of person-to-person interactions from distributed rfid sensor networks, PLOS ONE 5, e11596 (2010).

[42] J. Stehlé, A. Barrat, and G. Bianconi, Dynamical and bursty interactions in social networks, Phys. Rev. E 81, 035101(R) (2010).

[43] K. Zhao, J. Stehlé, G. Bianconi, and A. Barrat, Social network dynamics of face-to-face interactions, Phys. Rev. E 83, 056109 (2011).

[44] G. Cencetti, F. Battiston, B. Lepri, and M. Karsai, Temporal properties of higher-order interactions in social networks, arXiv , 2010.03404 (2020).

[45] C. Berge, Hypergraphs: Combinatorics of Finite Sets (North Holland, 1989).

[46] F. Battiston, G. Cencetti, I. Iacopini, V. Latora, M. Lucas, A. Patania, J.-G. Young, and G. Petri, Networks beyond pairwise interactions: Structure and dynamics, Phys. Rep. 874, 1 (2020).

[47] L. Torres, A. S. Blevins, D. S. Bassett, and T. Eliassi-Rad, The why, how, and when of representations for complex systems, arXiv , 2006.02870 (2020).

[48] M. Granovetter, Threshold Models of Collective Behavior, Am. J. Sociol. 83, 1420 (1978).

[49] D. J. Watts, A simple model of global cascades on random networks, Proc. Natl. Acad. Sci. U.S.A. 99, 5766 (2002).
[50] P. S. Dodds and D. J. Watts, Universal Behavior in a Generalized Model of Contagion, Phys. Rev. Lett. 92, 218701 (2004).

[51] J. Anttila, L. Mikonranta, T. Ketola, V. Kaitala, J. Laakso, and L. Ruokolainen, A mechanistic underpinning for sigmoid dosedependent infection, Oikos 126, 910 (2017).

[52] N. W. Landry and J. G. Restrepo, The effect of heterogeneity on hypergraph contagion models, Chaos 30, 103117 (2020).

[53] W.-m. Liu, H. W. Hethcote, and S. A. Levin, Dynamical behavior of epidemiological models with nonlinear incidence rates, J. Math. Biol. 25, 359 (1987).

[54] S. V. Scarpino, A. Allard, and L. Hébert-Dufresne, The effect of a prudent adaptive behaviour on disease transmission, Nat. Phys. 12, 1042 (2016).

[55] I. Iacopini, G. Petri, A. Barrat, and V. Latora, Simplicial models of social contagion, Nat. Commun. 10, 2485 (2019).

[56] B. Jhun, M. Jo, and B. Kahng, Simplicial SIS model in scalefree uniform hypergraph, J. Stat. Mech. 2019, 123207 (2019).

[57] G. F. de Arruda, G. Petri, and Y. Moreno, Social contagion models on hypergraphs, Phys. Rev. Research 2, 023032 (2020).

[58] L. Hébert-Dufresne, P.-A. Noël, V. Marceau, A. Allard, and L. J. Dubé, Propagation dynamics on networks featuring complex topologies, Phys. Rev. E 82, 036115 (2010).

[59] V. Marceau, P.-A. Noël, L. Hébert-Dufresne, A. Allard, and L. J. Dubé, Adaptive networks: Coevolution of disease and topology, Phys. Rev. E 82, 036116 (2010).

[60] J. P. Gleeson, High-accuracy approximation of binary-state dynamics on networks, Phys. Rev. Lett. 107, 068701 (2011).

[61] J. Lindquist, J. Ma, P. van den Driessche, and F. H. Willeboordse, Effective degree network disease models, J. Math. Biol. 62, 143 (2011).

[62] D. J. P. O’Sullivan, G. J. O'Keeffe, P. G. Fennell, and J. P. Gleeson, Mathematical modeling of complex contagion on clustered networks, Front. Phys. 3, 71 (2015).

[63] G. St-Onge, V. Thibeault, A. Allard, L. J. Dubé, and L. HébertDufresne, Social confinement and mesoscopic localization of epidemics on networks, Phys. Rev. Lett. 126, 098301 (2021).

[64] G. St-Onge, V. Thibeault, A. Allard, L. J. Dubé, and L. HébertDufresne, Master equation analysis of mesoscopic localization in contagion dynamics on higher-order networks, Phys. Rev. E 103, 032301 (2021).

[65] S. Nadarajah, Exact distribution of the product of $\mathrm{m}$ gamma and n Pareto random variables, J. Comput. Appl. Math. 235, 4496 (2011).

[66] B. K. Fosdick, D. B. Larremore, J. Nishimura, and J. Ugander, Configuring random graph models with fixed degree sequences, SIAM Rev. 60, 315 (2018).

[67] P. S. Chodrow, Configuration models of random hypergraphs, J. Complex Netw. 8, cnaa018 (2020).

[68] M. M. de Oliveira and R. Dickman, How to simulate the quasistationary state, Phys. Rev. E 71, 016129 (2005).

[69] G. St-Onge, H. Sun, A. Allard, L. Hébert-Dufresne, and G. Bianconi, Superspreading events data from arxiv:2101.07229 (2021), updated: 2021-02-01.

[70] G. St-Onge, H. Sun, A. Allard, L. Hébert-Dufresne, and G. Bianconi, heterogeneous-exposure-hons (2021). 